The Yellow Fever Research Institute is supported by the Rockefeller Foundation, the Governments of the British West African Colonies and the Colonial Medical Research Service.

Yellow Fever Research Institute,

LeONARD JaN ChWATt

Lagos-Yaba, Nigeria,

British West Africa.

Feb. 10.

'Dalziel, J. M., Bull. Ent. Re8., 11, 247 (1920-21). 'Dunn, I. H., Bull. Ent. Res., 17, 183 (1926); 18, 139 (1927); 18 ;

${ }^{3}$ Edwards, F. W., Bull. Ent. Res., 3, 1 (1912); "Mosquitoes of the Ethiopian Region", part III, "Culicine Adults and Pupæ", 130 (London: British Museum (Natural History), 1941).

\section{Overlapping of Dust Particles on a Sampling Plate}

IF, in a dust sample intended for microscopic examination, the number of particles per unit area were too large, some of the particles would overlap. Such 'clumps' would be counted as single particles instead of two, three or more, and the total count would be too low. The following analysis gives an indication of the magnitude of this effect. We shall assume that the particles may be regarded as circular laminæ of diameter $d$, and that their centres are distributed randomly over an area $A$. If the particles were of highly irregular shape and size, the present results might be invalid.

Any individual particle will be overlapped by $r-1$ other particles if $r-1$ centres fall within the circle with centre coinciding with the given particle centre, and radius $d$. If we assumed that in all clumps of order $r$, each of the $r$ particles overlapped all the others, the probability that a particle would belong to a clump of order $r$ would be (for large $N$ )

$$
\frac{\exp \left(-m^{\prime}\right)\left(m^{\prime}\right)^{r 1}}{(r-1) !}
$$

where $m^{\prime}=N \pi d^{2} / A, N / A$ being the mean number of centres per unit area.

If, on the average, $P_{r}$ clumps of order $r$ were observed (so that $\sum_{r=1}^{\infty} P_{r}=C$, the number of clumps counted, and $\left.\sum_{r=1}^{\infty} r P_{r}=N\right)$, we should have

$$
\begin{aligned}
r P_{r} & =\frac{N \exp \left(-m^{\prime}\right)(m)^{r-1}}{(r-1) !}, \\
\therefore \quad P_{r} & =\left(\frac{N \exp \left(-m^{\prime}\right)}{m^{\prime}}\right) \cdot \frac{\left(m^{\prime}\right)^{r}}{r !},
\end{aligned}
$$

so that the clump-size $r$ follows a curtailed Poisson distribution (with missing zero term).

If $m=N / C$ is the mean clump-size,

$$
m=\frac{N \pi d^{2} / A}{\mathrm{I}-\exp \left(-N \pi d^{2} / A\right)} .
$$

This analysis may be expected to be inexact when the frequency of clumps of more than two particles is appreciable. In such cases the frequencies $P_{r}$, for high values of $r$, are much higher than we should expect, owing to the existence of clumps in the form of chains, and the mean $m$ is also increased. As $N \pi d^{2} / A \rightarrow 0, m$ tends to the value given in (1). Sampling experiments with random numbers have shown that (1) appears to under-estimate the true value of $m$ by less than 10 per cent, even for values of $N d^{2} / A$ as high as $0 \cdot 3$. In practical work, concentrations should be considerably less than this.

For given values of $C, d$ and $A$, we may estimate $m$ by transforming equation (1) to the following :

$$
m=\frac{-A}{C \pi d^{2}} \log _{\epsilon}\left(1-\frac{C \pi d^{2}}{A}\right),
$$

$m^{\prime}$ (if required) being obtained from the equation

$$
m=\frac{m^{\prime}}{1-\exp \left(-m^{\prime}\right)} \text {. }
$$

In order to reduce the percentage error of overlapping, $100(N-C) / C$, to less than 5 per cent, we need $m<1.05$, which from (2) is equivalent to

$$
C<A / 10 \cdot 7 \pi d^{2}=0.0297 A / d^{2} \text {. }
$$

Statistical Research Unit,

J. O. IRWIN

P. Armitage

Group for Research in

C. N. Davies

Industrial Physiology,

(Medical Research Council),

London School of Hygiene and Tropical Medicine,

London, W.C.1.

\section{Dispersibility of Coal in a Supersonic Field}

IN the course of experiments on the solubility (or dispersibility) of coals in organic solvents, it was observed that the pyridine-insoluble $(\alpha)$ fraction of a Northumberland coal $(82.6$ per cent carbon and $5 \cdot 1$ per cent hydrogen on the dry ash-free basis) became partially dissolved (or dispersed) when suspended in pure dry pyridine and exposed to supersonic waves (c. $25 \mathrm{kc} / \mathrm{sec}$.) for $15 \mathrm{~min}$. When the extract thus formed was recovered by removal of the solvent under reduced pressure in an atmosphere of dry oxygen-free nitrogen, it formed a black, freerunning solid that was only very slightly soluble in boiling pyridine. The elementary composition of this material was practically identical with that of the original $\alpha$-fraction, both being reduced to the pure coal basis.

The same result was afterwards recorded with several other coals ranging from lignitous to bituminous; the starting material (that is, the $\alpha$-fraction) was in each case obtained by extracting the finely powdered coal with boiling pyridine in a Soxhlet apparatus for fourteen days.

These observations are of considerable interest. It has already been shown by Kiebler ${ }^{1}$ that the yield of extract obtainable from a given coal is statistically related to the internal pressure of the solvent. The effects here described appear to form an extension of this generalization : they imply that the quantity of material which can be extracted from a coal varies with the total energy that is available for the detachment of molecules (or micelles ${ }^{2}$ ) from the coal lump or particle. They lead, however, to an important additional conclusion : theoretically, the supersonic effect may be connected with one of three mechanisms. Thus one may suppose either (1) that supersonic waves effect a breakdown of large molecules contained in coal, or (2) that they cause a disruption of individual coal micelles, or (3) that they bring about a dispersion of micelles in their entirety. 